\title{
Holographic characterization of chain photopolymerization
}

\author{
D. Engin, ${ }^{*}$ A. S. Kewitsch, ${ }^{*}$ and A. Yariv \\ Department of Applied Physics, California Institute of Technology, 128-95, Pasadena, California 91125
}

Received September 29, 1998; revised manuscript received April 22, 1999

\begin{abstract}
A holographic characterization technique is developed in accordance with a general photopolymerization model. The technique allows detailed quantification of the chemical parameters, including their variation from the Trommsdorff effect. The holographic procedure is especially suited for studying the diffusion of the chemical reactants. (c) 1999 Optical Society of America [S0740-3224(99)01408-3]
\end{abstract}

OCIS codes: $090.2900,210.2860,190.4420,160.5470$.

\section{INTRODUCTION}

Photopolymerization has a wide range of applications, including lithography and optical data storage. Low material cost, large index changes, and high sensitivity are attractive aspects of these materials. Recently, various spatial nonlinear optical effects, such as self-trapping, have drawn attention to these chemicals. In Ref. 1, selftrapping is used to create high-aspect-ratio structures. A detailed understanding of the material response is necessary for studying the nonlinear spatial dynamical phenomena. In this paper a holographic characterization technique is developed for studying the chemical reactions that govern the changes in the refractive index upon polymerization. There are various techniques for monitoring polymerization dynamics. ${ }^{2-5}$ An all-optical characterization technique is favorable, since it directly studies the refractive-index changes, the quantity of interest in nonlinear optical phenomena.

Here a chain-polymerization model ${ }^{6}$ is taken as the underlying model, since the multifunctional acrylates are known to behave in this manner. ${ }^{2}$ In Section 2 the model is presented. Various versions of the same model have been used in studies of holographic data-storage materials. ${ }^{3}$ We assume a general form of the model, including the dependencies of the chemical constants on the degree of polymerization. This dependency is devised to model the Trommsdorff effect, ${ }^{6}$ which results in dramatic variations in the rate of change in the refractive index during the polymerization reaction owing to the changing properties of the chemical mixture (i.e., viscosity and molar density). The model also incorporates monomer diffusion, the strength of which may also change during the reaction. Monomer diffusion is essential for achieving fixed holograms in optical data storage, and it can be a limiting factor for the shape and the size of features possible in lithographic applications.

In Section 3 the hol ographic characterization technique is described. The technique studies the dynamics exhibited by a small grating under various illumination conditions. We are able to quantify all the chemical parameters, i.e., termination, propagation, and monomer diffusion constants, including their functional dependen- cies on the degree of polymerization. In Section 4 experiments are presented in which the characterization technique is carried out for various multifunctional acrylates.

\section{STARTING EQUATIONS}

Photoinduced chain polymerization ${ }^{6}$ is governed by the following kinetic equations:

$$
\begin{aligned}
\frac{d r}{d t} & =\Phi \mid s(0)-2 k_{t}(m) r^{2}, \\
\frac{d m}{d t}-D_{m}(m) \frac{d^{2} m}{d x^{2}} & =-k_{p}(m) m r \\
\frac{d \Delta n}{d t} & =\beta k_{p}(m) m r
\end{aligned}
$$

where $\mathrm{m}$ and $\mathrm{r}$ are the molar concentrations of monomers and radicals, respectively. The change in the refractive index, $\Delta \mathrm{n}$, is assumed to be proportional to the monomer concentration remaining in the solution (degree of polymerization), $\beta$ being the proportionality constant. Polymerization is initiated by the photoinitiators of concentration $\mathrm{s}$ in the monomer solution upon exposure to the incident light intensity I. Here, the depletion of the photoinitiators is assumed to be much slower than the polymerization reaction. This regime is convenient for the purposes of characterization and may be achieved by the choice of photoinitiators or by operation at low-enough intensities in the case of bimolecular termination. The photoinitiation coefficient $\Phi$ depends both on the quantum efficiency of the photocleavage process and the cross section of photoexcitation. ${ }^{4}$

The rate constants $k_{p}(m)$ and $k_{t}(m)$ describe chain propagation and chain termination by disproportionation (and coupling), respectively. The Trommsdorff effect is model ed by the dependence of these constants on the local monomer concentration. Diffusion of monomers is included in the model through the diffusion constant $D_{m}(m)$, which is also taken to be a function of the local monomer concentration. In accordance with holography in thin films a single spatial dimension $\mathrm{x}$ is considered. 
Here the induction period owing to oxygen scavenging ${ }^{5}$ is assumed not to affect the polymerization reaction. This requires proper isolation of the monomer sample from the air.

\section{CHARACTERIZATION TECHNIQUE AND LINEARIZED REGIME}

The characterization technique involves illumination of the monomer with a constant strong beam. A much weaker beam is turned on sometime after the induction period, for a short time (compared with the polymerization dynamics) during which a weak grating is written. The above scenario can be modeled by a set of ordinary differential equations that can be derived from the starting equations:

$$
\begin{aligned}
r_{0 s}\left(m_{0}\right)= & \sqrt{\frac{s_{0}(0) I_{0} \phi}{2 k_{t}\left(m_{0}\right)},} \\
\frac{d m_{0}}{d t}= & -k_{p}\left(m_{0}\right) r_{0 s}\left(m_{0}\right) m_{0}, \\
\Delta n_{0}= & \beta\left(m_{0}(0)-m_{0}\right), \\
\frac{d r_{1}}{d t}= & -4 k_{t}\left(m_{0}\right) r_{0 s}\left(m_{0}\right) r_{1}+\Phi I_{1} s_{0}(0), \\
\frac{d m_{1}}{d t}= & -\left\{D_{m}\left(m_{0}\right) \kappa^{2}+k_{p}\left(m_{0}\right) r_{0 s}\left(m_{0}\right)\right. \\
& \left.+\frac{d\left[k_{p}\left(m_{0}\right) r_{0 s}\left(m_{0}\right)\right]}{d m_{0}}\right\} m_{1}-k_{p}\left(m_{0}\right) r_{1} m_{0},
\end{aligned}
$$

$$
\begin{aligned}
\frac{\mathrm{d} \Delta \mathrm{n}_{1}}{\mathrm{dt}}= & \beta\left\{\mathrm{k}_{\mathrm{p}}\left(\mathrm{m}_{0}\right) \mathrm{r}_{0 \mathrm{~s}}\left(\mathrm{~m}_{0}\right)+\frac{\mathrm{d}\left[\mathrm{k}_{\mathrm{p}}\left(\mathrm{m}_{0}\right) \mathrm{r}_{0 \mathrm{~s}}\left(\mathrm{~m}_{0}\right)\right]}{\left.\mathrm{dm_{0 }}\right\}}\right. \\
& \times \mathrm{m}_{1}+\beta \mathrm{k}_{\mathrm{p}}\left(\mathrm{m}_{0}\right) \mathrm{r}_{1} \mathrm{~m}_{0},
\end{aligned}
$$

where $\mathrm{I}=\mathrm{I}_{0}+\mathrm{I}_{1}(\mathrm{t}) \exp (\mathrm{i} \kappa \mathrm{x})+\mathrm{C} . \mathrm{c}$. is the incident intensity distribution. The spatially varying component, $\mathrm{I}_{1}(\mathrm{t})$, is turned on for a short time, $\tau_{\text {on }}$. In the lowmodulation regime $\mathrm{I}_{0} \gg \mathrm{I}_{1}$, the first few Fourier amplitudes of the chemical concentrations are sufficient to describe the system:

$$
\begin{gathered}
r=r_{0 s}+r_{1} \exp (i \kappa x)+c . c ., \\
m=m_{0}+m_{1} \exp (i \kappa x)+c . c .,
\end{gathered}
$$

where $r_{1}$ and $m_{1}$ are the complex amplitude of the radical and monomer concentration components with spatial frequency $\kappa$. Similarly we define the complex-valued amplitudes $\Delta \mathrm{n}_{0}$ and $\Delta \mathrm{n}_{1}$.

Zero-order amplitudes follow simple dynamics. Very early on in the dynamics the average radical concentration achieves quasi steady state, $r_{0 s}\left(m_{0}\right)$. The presence of the radicals facilitates the depletion of the monomers with the polymerization rate $1 /\left[\tau_{\mathrm{p}}\left(\mathrm{m}_{0}\right)\right]$ $=\mathrm{k}_{\mathrm{p}}\left(\mathrm{m}_{0}\right) \mathrm{r}_{0 \mathrm{~s}}\left(\mathrm{~m}_{0}\right)$ [Fig. 1(a)]. During polymerization the polymerization rate changes adiabatically with the slowly changing monomer concentration owing to the Tromms- dorff effect; as a result the monomer-depletion dynamics deviate from a simple exponential decay. Simulations in Figs. 1 and 2 are carried out with the chemical parameters listed in Table 1.

Dynamics of the first-order amplitudes strongly depend on the monomer diffusion. For the purposes of characterization it is convenient to treat the monomer-diffusiondominated regime separately. Figures 1, 2(a), and 2(b) show the typical dynamics observed in the absence of monomer diffusion. In an experiment this regime can be achieved by choice of a high-enough average intensity, $I_{0}$. The condition $1 /\left[\tau_{\mathrm{p}}\left(\mathrm{m}_{0}\right)\right] \gg \mathrm{D}_{\mathrm{m}}\left(\mathrm{m}_{0}\right) \kappa^{2}$ is a sufficient condition.

When the second beam is turned on, the spatially varying radical concentration, $r_{1}$, achieves a quasi-steadystate value $r_{1 s}\left(m_{0}\right)=-I_{1} s_{0}(0) \phi /\left[4 k_{t}\left(m_{0}\right) r_{0 s}\left(m_{0}\right)\right]$ quickly [Fig. 2(a)], with time constant $\tau_{\mathrm{r}}=1$ / $\left[4 \mathrm{k}_{\mathrm{t}}\left(\mathrm{m}_{0}\right) \mathrm{r}_{0 \mathrm{~s}}\left(\mathrm{~m}_{0}\right)\right]$. Note that $\tau_{\mathrm{r}} \ll \tau_{\mathrm{p}}$; thus $\mathrm{m}_{0}$ can be taken to be a constant during the radical dynamics. Buildup of a spatially varying radical concentration drives the formation of $m_{1}$ and $\Delta n_{1}$. The grating amplitude $\Delta n_{1}$ in phase with $I_{1}$ grows and achieves roughly an amplitude of $\Delta \mathrm{n}_{1 \mathrm{~m}} \approx \beta \mathrm{k}_{\mathrm{p}}\left(\mathrm{m}_{0}\right) \mathrm{r}_{1 \mathrm{~s}}\left(\mathrm{~m}_{0}\right) \tau_{\mathrm{on}}$, as shown in Fig. 2(b). When the second beam is turned off, $r_{1}$ quickly decays with time constant $\tau_{r}$. As a result, the growth of $\Delta \mathrm{n}_{1}$ slows down, and the grating amplitude peaks shortly after $\tau_{\text {on }}$ at time $\tau_{\mathrm{r}}{ }^{\prime}\left(\mathrm{m}_{0}\right)=\tau_{\mathrm{r}} \ln \left\{\left[\tau_{\mathrm{p}}\left(\mathrm{m}_{0}\right)\right] / \tau_{\text {on }}\right\}$ [Fig. 2(c)]. After the decay of $r_{1}$, a gradual decay of the grating to zero takes place [Fig. 2(c)]. Setting $r_{1}$ and $r_{0}$ to zero in Eqs. (2a)-(2f), we can show that the rate of change of the grating amplitude, $\Delta \mathrm{n}_{1}$, is proportional to $\mathrm{m}_{0} / \tau_{\mathrm{p}}\left(\mathrm{m}_{0}\right)$, which is the rate of change of $m_{0}$. From this observation

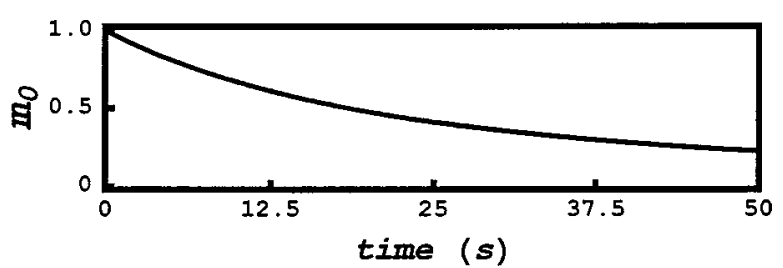

(a)

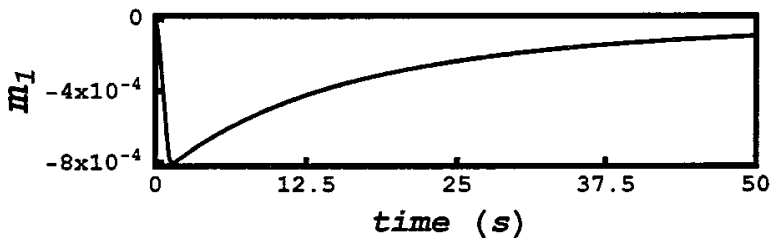

(b)

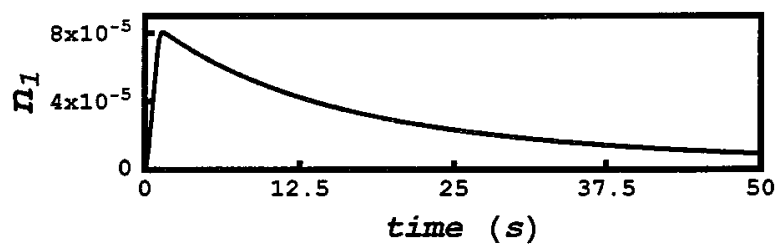

(c)

Fig. 1. Slow dynamics exhibited by (a) the average monomer concentration, (b) the spatially varying monomer concentration, and (c) the grating amplitude. The grating is gradually erased under constant illumination. The decay deviates from a simple exponential behavior because of the Trommsdorff effect. 


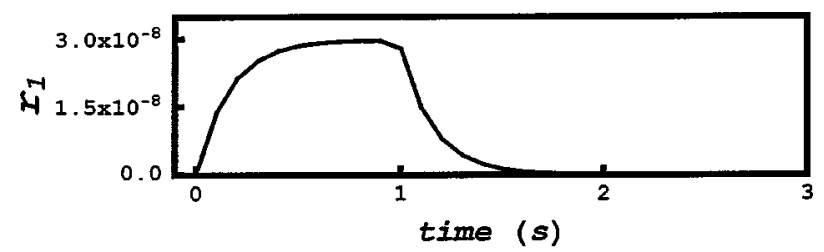

(a)

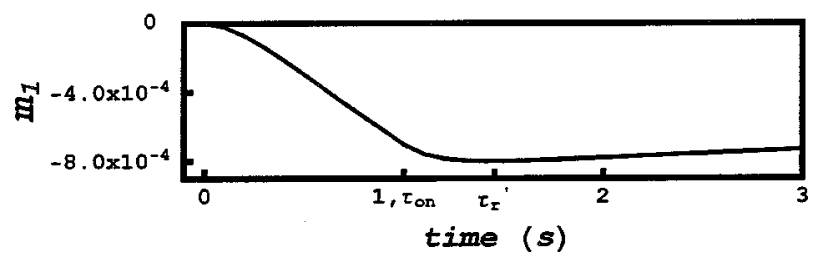

(b)

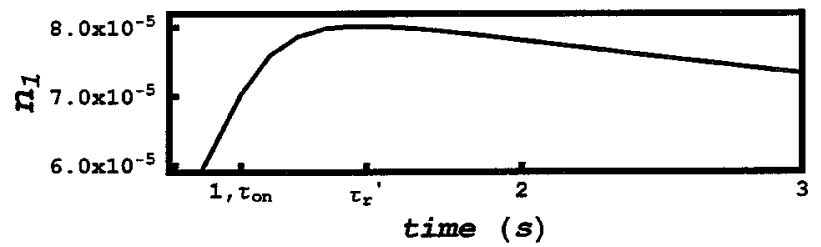

(c)

Fig. 2. Fast dynamics exhibited by the spatially varying amplitudes of (a) radical concentration, (b) monomer concentration, and (c) refractive-index change during and immediately after the grating writing stage. Grating writing is stopped at time $\tau_{\text {on }}$, and the grating amplitude peaks after a time of $\tau_{\mathrm{r}}{ }^{\prime}$.

Table 1. Values and Functional Forms of Kinetic Parameters Used in Numerical Simulations

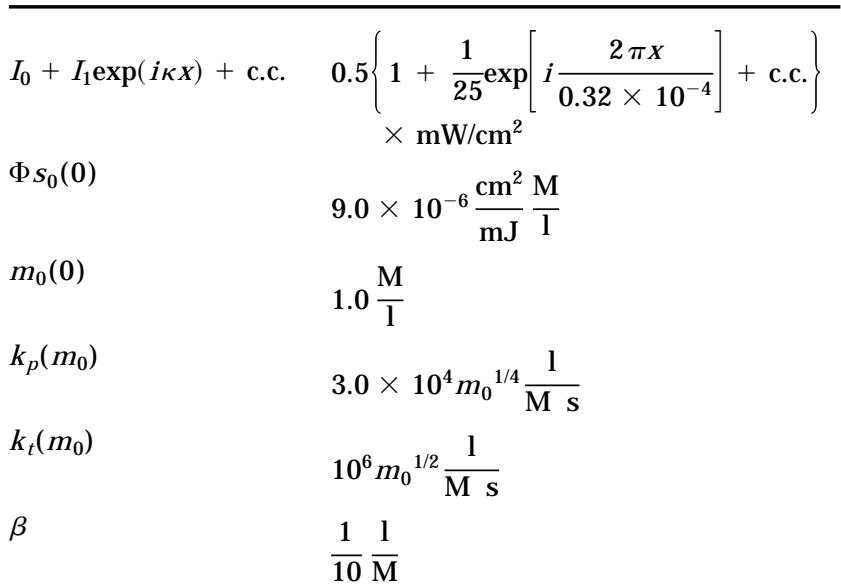

and knowing the initial monomer concentration, one can construct the average monomer dynamics from the measured decay of the grating amplitude, $\Delta \mathrm{n}_{1}$.

$$
\mathrm{m}_{0}(\mathrm{t})=\frac{\mathrm{m}_{0}(0) \int_{\mathrm{t}}^{\infty} \Delta \mathrm{n}_{1}\left(\mathrm{t}^{\prime}\right) d \mathrm{t}^{\prime}}{\int_{0}^{\infty} \Delta \mathrm{n}_{1}\left(\mathrm{t}^{\prime}\right) d \mathrm{t}^{\prime}}
$$

where the zero time corresponds to the beginning of the polymerization reaction (end of the induction period). In a similar manner one can construct $\tau_{\mathrm{p}}\left(\mathrm{m}_{0}\right)$ :

$$
\frac{1}{\tau_{\mathrm{p}}\left[\mathrm{m}_{0}(\mathrm{t})\right]}=\frac{\Delta \mathrm{n}_{1}(\mathrm{t})}{\int_{\mathrm{t}}^{\infty} \Delta \mathrm{n}_{1}\left(\mathrm{t}^{\prime}\right) d \mathrm{t}^{\prime}} .
$$

Notice that the above two constructions do not require knowledge of the multiplicative constants, such as $\beta$ or the thickness of hologram. This aspect is useful in realizing the above constructions from the experimental data. Figure 3 shows the construction carried out for the simulations shown in Figs. 1 and 2. The agreement between the constructed (solid curves) and the actual functions is good for the majority of the dynamics. The disagreement in Fig. 3(a) at long times arises from finite measurement time. The time, $\tau_{1}$, at which the difference starts becoming substantial [Fig. 3(a)] can be estimated from $\Delta \mathrm{n}_{1}\left(\tau_{1}\right)=10 \Delta \mathrm{n}_{1}\left(\tau_{\mathrm{m}}\right)$, where $\tau_{\mathrm{m}}$ is total measurement time. In an experiment the measurement time is limited by the noise floor, $\Delta \mathrm{n}_{1 \text { noise }}$, and $\Delta \mathrm{n}_{1}\left(\tau_{\mathrm{m}}\right)=\Delta \mathrm{n}_{\text {1noise }}$. In Fig. 3(b), finite measurement time manifests itself as an overestimation of the polymerization rate for small monomer concentrations. From the constructed $m_{0}$ one can determine the average monomer concentration near which the construction begins to fail: $\mathrm{m}_{0 \mathrm{l}}=\mathrm{m}_{0}\left(\tau_{1}\right)$ [Fig. $3(b)$ ]. Early on in the polymerization dynamics the constructed functions also deviate from the actual functions owing to the finite time it takes for the grating buildup. For times larger than $\tau_{\mathrm{u}}=3 \tau_{\mathrm{r}}{ }^{\prime}$ we find that the above construction works well. We expect the results to be accurate for $m_{01}<m_{0}<m_{0 u}$, as depicted in Fig. 3(b).

The chemical constants $k_{p}\left(m_{0}\right)$ and $k_{t}\left(m_{0}\right)$ are determined by measurement of $\tau_{\mathrm{p}}\left(\mathrm{m}_{0}\right)$ and $\tau_{\mathrm{r}}{ }^{\prime}\left(\mathrm{m}_{0}\right)$ at particular points of the polymerization reaction. The polymerization rate, $1 / \tau_{\mathrm{p}}\left(\mathrm{m}_{0}\right)$, can be determined by a single measurement when the grating is written right after the induction period. On the other hand, $\tau_{\mathrm{r}}{ }^{\prime}\left(\mathrm{m}_{0}\right)$ is deter-

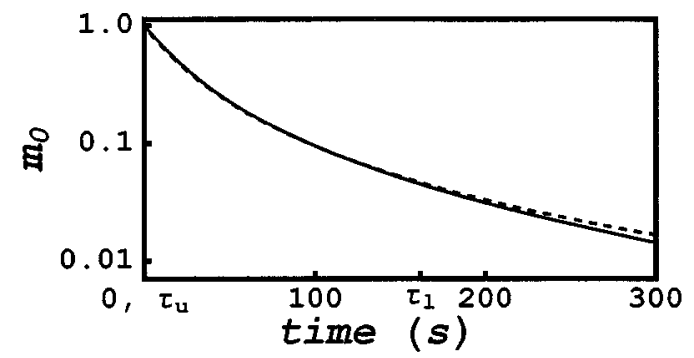

(a)

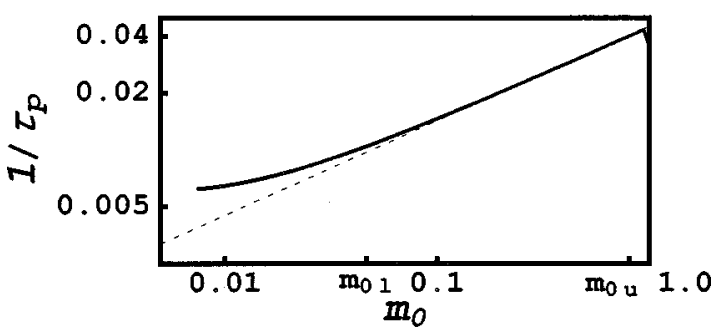

(b)

Fig. 3. Comparison of the constructed (solid curve) and actual (dashed curve) chemical parameters: (a) average monomer concentration dynamics (log-linear); (b) monomer concentration dependence of the polymerization rate (log-log). The constructions are expected to be accurate for $\mathrm{m}_{01}<\mathrm{m}_{0}<\mathrm{m}_{0 \mathrm{u}}$. 


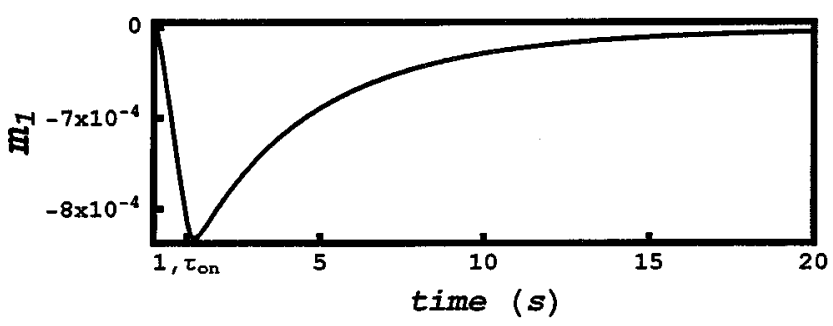

(a)

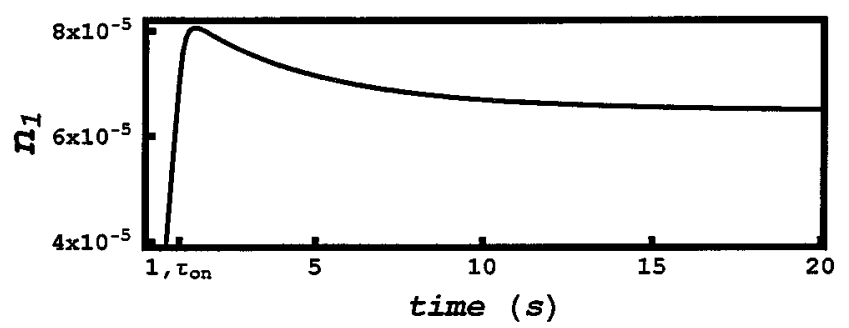

(b)

Fig. 4. Dynamics in the diffusion-dominated regime: (a) spatially varying monomer concentration quickly decays owing to diffusion; (b) at the same rate the grating amplitude, $\Delta n_{1}$, achieves a steady, fixed amplitude.

mined by multiple measurements when the grating is written at different points of the polymerization reaction.

One interesting signature of this regime is scaling of both radical and monomer dynamics of $\sqrt{I_{0}}$. The scaling can be realized in Eqs. (2) by a coordinate transformation as $\mathrm{t} \rightarrow \mathrm{t}^{\prime}\left(\mathrm{I}_{0}{ }^{\prime} / I_{0}\right)^{1 / 2}$, where $\mathrm{I}_{0}{ }^{\prime}$ is an arbitrary reference intensity level. The scaling for $m_{0}$ dynamics follows from the proportionality of the polymerization rate $1 / \tau_{\mathrm{p}}\left(\mathrm{m}_{0}\right)$ to $r_{0 s}$. The scaling for $m_{1}$ only works in the regime where the monomer diffusion can be neglected. The proportionality of the polymerization rate and of the quasi-steadystate concentration $r_{1 s}$ to $\sqrt{I_{0}}$ facilitates the scaling in all stages of the characterization scheme. We utilized the scaling properties of the dynamics to construct $m_{0}$ dependency of the monomer-diffusion constant, $\mathrm{D}_{\mathrm{m}}\left(\mathrm{m}_{0}\right)$.

Figure 4 shows the dynamics exhibited in the diffusiondominated regime. The diffusion constant is taken to be $D_{m}\left(m_{0}\right)=6 \times 10^{-12} m_{0}$, and all the other parameters are the same as in Table 1. The main difference in the presence of diffusion is the formation of a fixed grating at the end of the process. For the point of characterization it is convenient to measure the monomer-diffusion constant when the diffusion rate satisfies $\tau_{\mathrm{r}} \ll 1 / \mathrm{D}_{\mathrm{m}} \tau^{2}$ $\ll \tau_{\mathrm{p}}$. Starting from the high-intensity case in which the polymerization dynamics is fast compared with the monomer diffusion, one can achieve this condition by gradually decreasing the average intensity. In this regime grating, amplitude again achieves roughly a value of $\Delta \mathrm{n}_{1 \mathrm{~m}} \approx \beta \mathrm{k}_{\mathrm{p}}\left(\mathrm{m}_{0}\right) \mathrm{r}_{1 \mathrm{~s}}\left(\mathrm{~m}_{0}\right) \tau_{\text {on }}$ while the second beam is on. However, after the beam is turned off, spatially varying monomer concentration $\mathrm{m}_{1}$ decays with the monomerdiffusion rate instead of the polymerization rate to zero. As a consequence, the grating amplitude decays at the diffusion rate to a final fixed value of $\Delta \mathrm{n}_{1 \mathrm{f}} \approx \Delta \mathrm{n}_{1 \mathrm{~m}}[1$ - $\left.1 / D_{m}\left(m_{0}\right) \kappa^{2} \tau_{p}\right]$. Experimentally, a 1:10 ratio between the diffusion rate and the polymerization rate would correspond to a measurable $10 \%$ decay in the grating amplitude. In this regime, dependency of the diffu- sion constant on the local monomer concentration can be determined by carrying out multiple measurements when the grating is written at different points of the polymerization reaction [as in determining $\tau_{\mathrm{r}}{ }^{\prime}\left(\mathrm{m}_{0}\right)$ ].

Finally a simple technique for determining an order of magnitude value for the monomer-diffusion constant is described. In chemicals where the monomer diffusion is small, as is the case for chemicals examined in this paper, the above technique may require long times for gratingdecay measurements. A simpler way to look for monomer diffusion is to turn off both the strong and the weak beams after writing a weak grating. In this case, radicals quickly disappear, and the only way for the spatially varying monomer concentration to decay is through diffusion. The chemical is kept in the dark for some time $\tau_{\text {dark }}$, after which the strong beam is turned on. If the monomer diffusion time is less than or comparable to the dark time, $D_{m} \kappa^{2} \geqslant 1 / \tau_{\text {dark }}$, then the grating does not disappear completely upon exposure to a single strong beam. Thus by observing the presence of a fixed grating after the uniform illumination, one can acquire an order of magnitude estimation of the monomer diffusion.

\section{EXPERIMENTS}

In this section we describe photopolymerization experiments carried out with a multifunctional monomer, TMPTA from UCB Radcure. I rgacure 369 from CibaGeigy is used as the photoinitiator. A monomerphotoinitiator solution is prepared with a $0.1 \mathrm{wt} . \%$ photoinitiator concentration. From this an initial initiator molar concentration of $\mathrm{s}_{0}(0)=3 \times 10^{-3} \mathrm{M} / \mathrm{l}$ can be calculated. Irgacure 369 exhibits bleaching upon photocleavage, ${ }^{7}$ and thus a transmission experiment (at $325 \mathrm{~nm}$ from He-Cd laser) is carried out in order to determine the decay rate of the photoinitiators delta $I_{0} \Phi$. The monomer mixture is placed between two glass plates (no spacer is used). A monomer film thickness of typically $30-20 \mu \mathrm{m}$ is obtained. Approximately a $5 \%$ change in transmission is observed upon photopolymerization, from which the absorption within the polymer film is concluded to be small. The transmission experiment gives a value of $\Phi=0.003 \mathrm{~cm}^{3} / \mathrm{mJ}$, which implies a much slower (an order of magnitude) decay rate for the photoinitiators than the typical polymerization rate.

The holography setup used in the experiments is shown in Fig. 5. Two coherent, expanded (collimate diameter 5 $\mathrm{mm}$ ), extraordinarily polarized writing beams (at $325 \mathrm{~nm}$ ) are incident on the polymer film. The intensity ratio of the two writing beams is chosen to be $25: 1$, which guarantees low-intensity modulation and a constant average intensity. The angle between the two writing beams is $40^{\circ}$, which corresponds to a grating period of $0.32 \mu \mathrm{m}$. A computer-controlled shutter is placed in the optical path of the weaker writing beam. The grating is monitored by a phase-matched beam at $632 \mathrm{~nm}$, which does not initiate any photopolymerization. The diffracted red beam is measured with a photodiode, and the dynamics are re corded with a computer. U pon photopolymerization the phase-matching angle for the red beam is confirmed to remain the same. For small diffraction efficiencies, which is the case for these characterization experiments, no 


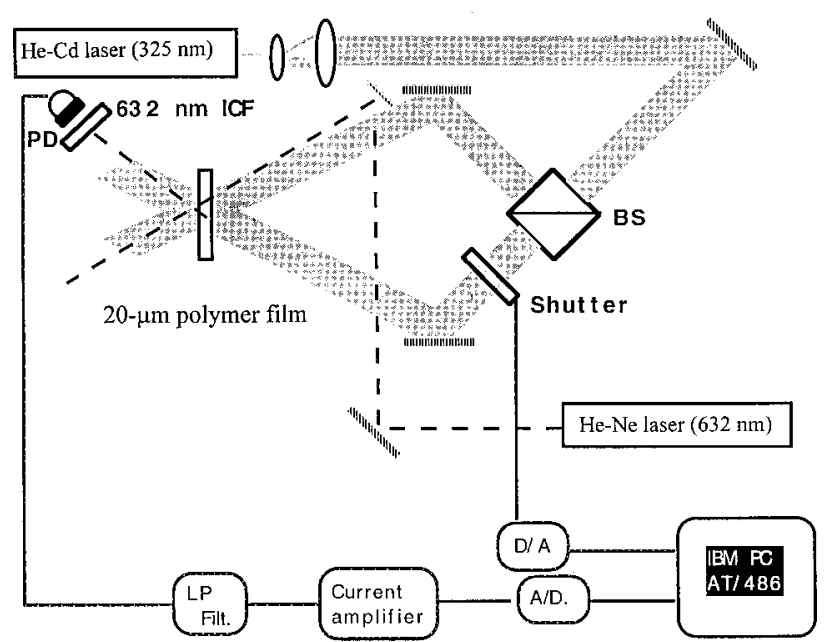

Fig. 5. Setup for the holography experiments: PD, photodiode; ICF , chromatic filter; BS, beam splitter; LP Filt., low-pass filter: $D / A$, digital-to-analog; $A / D$, analog-to-digital.

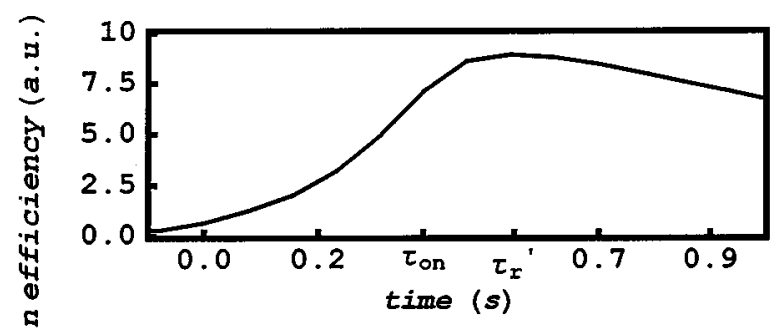

(a)

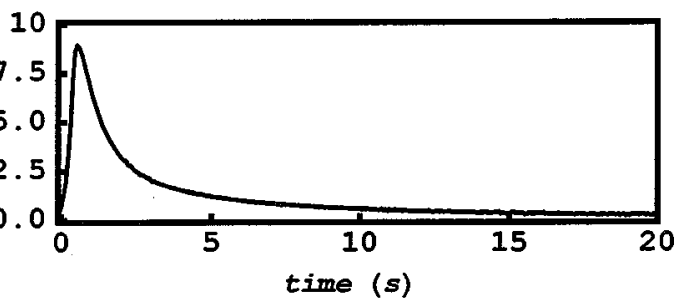

(b)

Fig. 6. Dynamics exhibited by the grating amplitude in the experiments: (a) during and immediately after the grating writing (time $\leqslant \tau_{\text {on }}$ ); (b) gradual decay of the grating under constant illumination. The decay does not follow an exponential law.

significant distortion of the reading beams owing to nonuniform photopolymerization is observed.

In Fig. 6, typical dynamics exhibited by the square root of the diffraction efficiency is shown for an average intensity of $1 \mathrm{~mW} / \mathrm{cm}^{2}$. After a period of no polymerization owing to oxygen scavenging, a sharp threshold is observed. The fast increase in diffraction efficiency as described in the previous section is caused by fast radical dynamics. After a buildup of a small grating ( $1 \%$ diffraction efficiency), the weak beam is turned off at $\tau_{\text {on }}$. Subsequently the growth of the grating amplitude slows down and peaks shortly after at time $\tau_{\mathrm{r}}$ [Fig. 6(a)]. Then a slow decay at the polymerization rate follows, as shown in Fig. 6(b). The decay of the grating does not follow a simple exponential function. The increasing viscosity of the material as the polymerization proceeds (Trommsdorff effect) results in the slowing down of the polymerization rate.
Since the diffraction efficiency is erased completely in Fig. 6(b), we conclude that the diffusion of monomers is not observable in this regime. We can carry out the construction of $\mathrm{m}_{0}$ and $\tau_{\mathrm{p}}\left(\mathrm{m}_{0}\right)$ as described in Eqs. (4) and (5). The results of the construction is shown in Fig. 7. From the considerations of the noise floor and the finite grating buildup time, $\tau_{\mathrm{r}}{ }^{\prime}\left[\mathrm{m}_{0}(0)\right]$, we expect the construction to be accurate for $0.4<\mathrm{m}_{0}<0.8$. From Fig. 7(a) an initial value for $\tau_{\mathrm{r}}{ }^{\prime}\left[\mathrm{m}_{0}(0)\right]$ is determined to be $200 \mathrm{~ms}$. From these two measurements we calculate the initial values of $k_{p}\left[m_{0}(0)\right]$ and $k_{t}\left[m_{0}(0)\right]$ to be $2 \times 10^{5}$ and $10^{6}$ I/(M s), respectively.

Next, the above measurement is carried out for various intensities ranging from 1 to $10^{-2} \mathrm{~mW} / \mathrm{cm}^{2}$. In Fig. 8 the square roots of the diffraction efficiencies for seven different intensities are shown in logarithmic scale. The linear transformation $\mathrm{t} \rightarrow \mathrm{t}^{\prime}\left(\mathrm{I}_{0}{ }^{\prime} / \mathrm{I}_{0}\right)^{1 / 2}$ is carried out, where the reference intensity $I_{0}{ }^{\prime}$ is equal to $1 \mathrm{~mW} / \mathrm{cm}^{2}$. Clearly for the most part of the dynamics, scaling works extremely well. This is a strong proof of the bimolecular termination reaction that is expected to take place in these chemicals. It also agrees with the assumption that the kinetic constants are only functions of the local monomer concentration. In Fig. 8(a) the dynamics right after the writing is shown. The time it takes for the diffraction efficiency to peak signifies the radical dynamics $\tau_{r}{ }^{\prime}$, and, as shown, the scaling works for the radical dynamics as well. Note that in these figures the quantity of interest is the slope of the logarithmic decay curves. Parameters that affect the final results as multiplicative constants (such as film thickness, proportionality constants between index change, and monomer concentration $\beta$ ) are expected to result in vertical shifts of the curves. In Fig. 8 , such shifts are accounted for by alignment of the decay curves at long times.

Lastly, the technique to determine an order of magnitude for the monomer diffusion is carried out in two other difunctional acrylate monomers, HDODA (UCB Radcure)

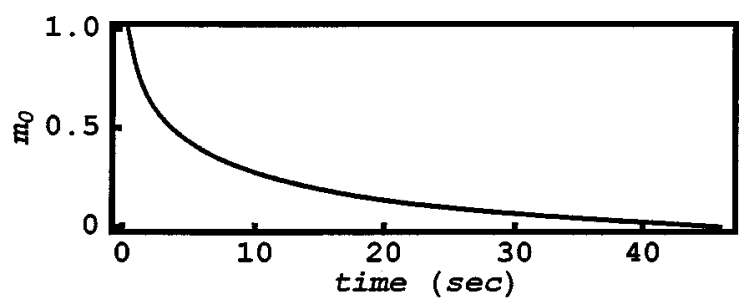

(a)

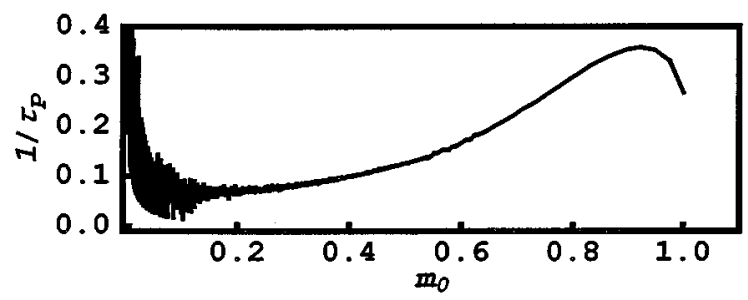

(b)

Fig. 7. Constructions of (a) average monomer concentration dynamics and (b) average monomer concentration dependence of the polymerization rate. Constructions are carried out with the experimental data shown in Fig. 6 . The results are expected to be accurate for $0.4 \leqslant m_{0} \leqslant 0.8$. 


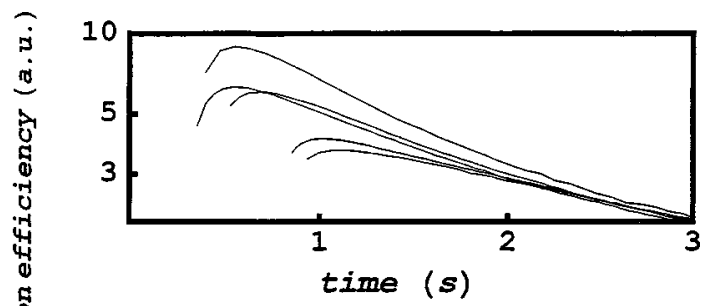

(a)

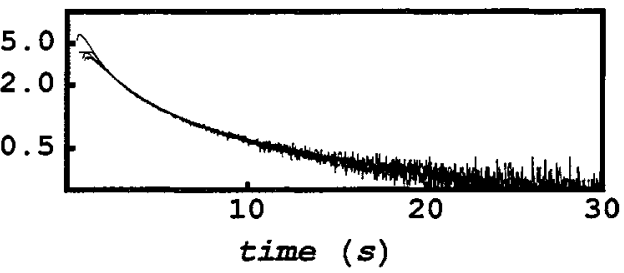

(b)

Fig. 8. Scaling of (a) the radical dynamics and (b) the monomer dynamics. The plots are shown in log-linear format.

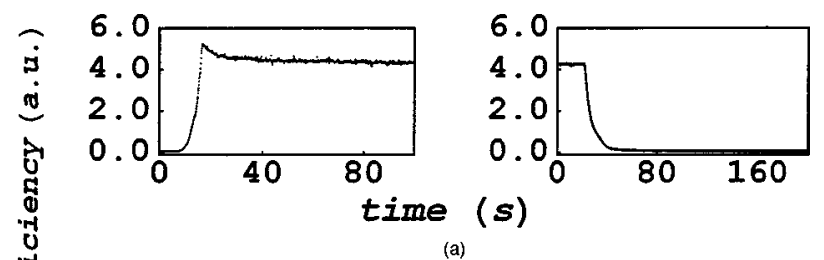

(a)

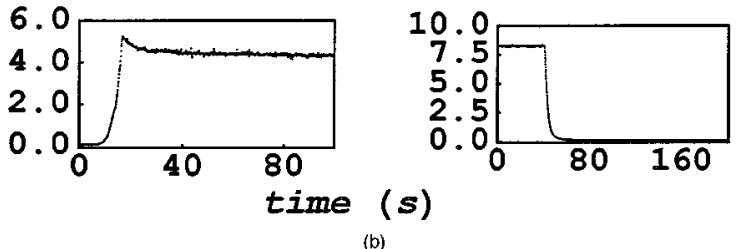

(b)

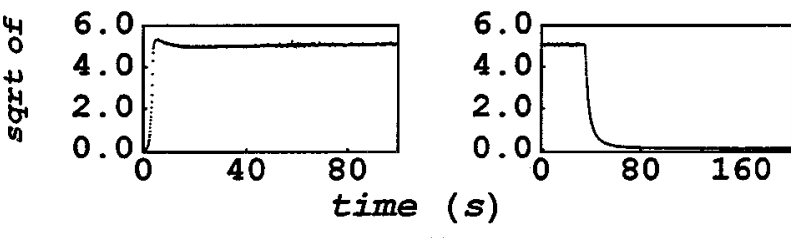

(c)

Fig. 9. Simple procedure to determine the order of magnitude of monomer diffusion is carried out in three different multifunctional acrylates: (a) TMTA, (b) butyle ethylene and (c) HDODA with 0.1 wt.\% initiator concentration (Irgacure 369). The dark waiting period is 30 minutes. Complete decay of the gratings after the dark period implies an exceptionally low diffusion constant.

and ethylene diacrylate with 0.1 wt.\% I rgacure 369 . The dark time in each experiment was $30 \mathrm{~min}$. As shown in Fig. 9 for each of the multifunctional monomers, the grating very quickly decays to zero when the erasure beam is turned on, signifying that the monomer diffusion is small. Considering the amount of time that was waited in the dark, one can calculate an upper limit for diffusion constants in these materials, $D_{\mathrm{m}} \ll 10^{-12} \mathrm{~cm}^{2} / \mathrm{s}$.

This is a surprising result considering that typical monomer-diffusion constants are of the order of $10^{-7}-10^{-9} \mathrm{~cm}^{2} / \mathrm{s}^{3}$ This may be due to the multifunctional nature of the monomers that were used. Multifunctionality of the monomer results in strong cross link- ing, which forms a polymer network early on in the polymerization dynamics, and is expected to result in considerable decrease in monomer diffusion. Also, since each molecule has in principle two or three functional groups, once one of the monomers (a functional group) reacts with a propagating radical, the remaining unreacted monomers on the molecule become part of a larger molecule (although they have not yet reacted). From this argument, by the time $10 \%$ conversion is completed, $30 \%$ $40 \%$ of the remaining monomers may al ready be part of a larger molecule and may not be able to diffuse.

\section{CONCLUSIONS AND SUMMARY}

The holographic characterization technique is simulated with a general photopolymerization model. The model accounts for the Trommsdorff effect through dependence of the kinetic parameters on the local degree of polymerization. Under such an assumption a proper scaling of the dynamics is predicted and shown to exist in the experiments. The sole dependence on the local monomer concentration makes it possible to retrieve the kinetic parameters as functions of the monomer concentration. The construction carried from experiments exhibits a dramatic slowing down of the dynamics during the polymerization reaction. The presence of monomer diffusion strongly influences the final state of the test grating. A suitable average intensity range is suggested for proper quantification of the diffusion constant. The scaling of the dynamics enables one to determine the influence of the Trommsdorff effect on the diffusion constant. Finally a very simple technique is suggested for acquiring an order-of-magnitude estimate of the diffusion constant. Using this technique, we are able to determine a minuscule upper limit for the diffusion constant in the multifunctional acrylates.

\section{ACKNOWLEDGMENTS}

This research was supported by the Advanced Research Projects Agency and the Office of Naval Research under Grant No. N0001492 1891. We thank B. Grubbs at Caltech for illuminating discussions about polymerization dynamics.

*Present address: Arroyo Optics, Incorporated, 1646 17th Street, Santa Monica, California 90404.

\section{REFERENCES}

1. A. S. Kewitsch and A. Yariv, "Nonlinear-optical properties of photoresists for projection lithography," Appl. Phys. Lett. 68, 455-457 (1996); A. S. Kewitsch and A. Yariv, "Selffocusing and self-trapping of optical beams upon photopolymerization," Opt. Lett. 21, 24-26 (1996).

2. C. Decker and K. Moussa, "Real-time kinetic study of laserinduced polymerization," Macromolecules 22, 4455-4462 (1989); C. Decker, "Photoinitiated curing of multifunctional monomers," Chimia 47(10), 378-382 (1993); C. Decker, "Recent advances in laser-induced curing," Radiation Curing 182 (4310), 383-386 (1992). 
3. V. V. Krongauz, in Processes in Photoreactive Polymers, V. V. Krongauz and A. D. Trifunac, eds. (Chapman and Hall, New York, 1995), Chaps. 2-5; S. Piazzolla and B. K. J enkins, "Holographic grating formation in photopolymers," Opt. Lett. 21, 1075-1077 (1996).

4. C. Decker, in Handbook of Polymer Science and Technol ogy, N. P. Cheremisinoff, ed. (Marcel Dekker, New York, 1989).
5. C. Decker, "Real-time monitoring of polymerization quantum yields," Macromolecules 23, 5217-5220 (1990).

6. G. Odion, Principles of Polymerization (Wiley, New York, 1981), Chap. 3

7. C. Decker, in Processes in Photoreactive Polymers, V. V. Krongauz and A. D. Trifunac, eds. (Chapman and Hall, New York, 1995), Chaps. 1 and 2. 